\title{
Edge-odd Gracefulness of the Graph $S_{2} \square S_{n}$
}

\author{
Dr. A. Solairaju \\ Associate Professor of Mathematics \\ Jamal Mohamed College, Tiruchirapalli - 620020. \\ Tamil Nadu, India. \\ C. Vimala and A.Sasikala \\ Assistant Professors (SG), Department of Mathematics, \\ Periyar Maniammai University, Vallam \\ Thanjavur - Post. Tamil Nadu, India.
}

\begin{abstract}
A (p, q) connected graph is edge-odd graceful graph if there exists an injective map $\quad \mathrm{f:} E(\mathrm{G}) \rightarrow\{1,3$, $\ldots, 2 \mathrm{q}-1\}$ so that induced map $\quad \mathrm{f}_{+}: \mathrm{V}(\mathrm{G}) \rightarrow\{0$, $1,2,3, \ldots,(2 \mathrm{k}-1)\}$ defined by $\quad \mathrm{f}_{+}(\mathrm{x}) \equiv \Sigma \mathrm{f}(\mathrm{x}, \mathrm{y})(\mathrm{mod}$ $2 \mathrm{k}$ ), where the vertex $\mathrm{x}$ is incident with other vertex $\mathrm{y}$ and $\mathrm{k}$ $=\max \{\mathrm{p}, \mathrm{q}\}$ makes all the edges distinct and odd. In this article, the Edge-odd gracefulness of $S_{2} \square S_{n}$ is obtained.
\end{abstract}

Keywords: Graceful Graph, Edge-odd graceful labeling, Edge-odd graceful graph

\section{INTRODUCTION}

A.Solairaju and K.Chitra [2009] obtained edge-odd graceful labeling of some graphs related to paths. A. Solairaju et.al. [2010] that the cartesian product of $P_{2}$ and $C_{n}$ for all integer $n$, and $S_{m, n}$.

Section-2: Edge-odd Gracefulness of book graph $S_{2} \square S_{n}$

Definition 2.1: Graceful Graph: A function $f$ of a graph $G$ is called a graceful labeling with $\mathrm{m}$ edges, if $\mathrm{f}$ is an injection from the vertex set of $\mathrm{G}$ to the set $\{0,1,2, \ldots, \mathrm{m}\}$ such that when each edge uv is assigned the label $\mid \mathrm{f}(\mathrm{u})-$ $\mathrm{f}(\mathrm{v}) \mid$ and the resulting edge labels are distinct. Then the graph $\mathrm{G}$ is graceful.

Definition 2.2: The book graph $S_{2} \square S_{n}$ is a connected graph obtained by adding ' $n$ ' number of $\mathrm{C}_{4}$ with one edge. It has $2 n$ vertices and $3 n-2$ edges. This graph is given in figure 1.

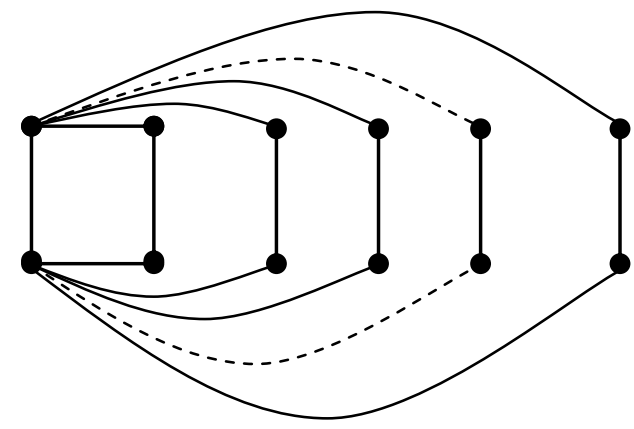

Figure 1: Graph of $S_{2} \square S_{n}$
Definition 2.2: Edge-odd graceful graph: A (p, q) connected graph is edge-odd graceful graph if there exists an injective map $\mathrm{f:} E(\mathrm{G}) \rightarrow\{1,3, \ldots, 2 \mathrm{q}-1\}$ so that induced map $\mathrm{f}_{+}: \mathrm{V}(\mathrm{G}) \rightarrow\{0,1,2, \ldots,(2 \mathrm{k}-1)\}$ defined by $\mathrm{f}_{+}(\mathrm{x}) \equiv \Sigma$ $\mathrm{f}(\mathrm{x}, \mathrm{y})(\bmod 2 \mathrm{k})$, where the vertex $\mathrm{x}$ is incident with other vertex $\mathrm{y}$ and $\mathrm{k}=\max \{\mathrm{p}, \mathrm{q}\}$ makes all the edges distinct and odd. Hence the graph $\mathrm{G}$ is edge- odd graceful.

Theorem 2.1: The connected graph $S_{2} \square S_{n}$, for $n \geq 3$, is edge - odd graceful.

Proof: The figure 2 is the Cartesian product graph $S_{2} \square S_{n}$ with $2 n$ vertices and $3 n-2$ edges, with some arbitrary labeling to its vertices and edges. It is proved that the graph $S_{2} \square S_{n}$, for $n \geq 3$, is edge - odd graceful by taking two cases such as $n$ is odd and $n$ is even.

Case (i) : $\mathrm{n}$ is odd

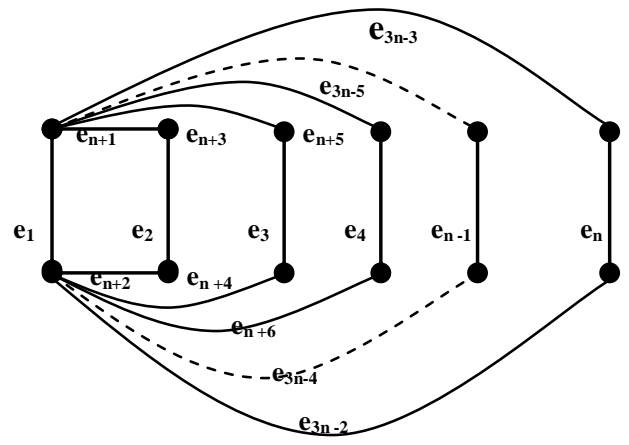

Figure 2: Edge - odd graceful Graph $S_{2} \square S_{n}$ for $n$ is odd

Hence define $\mathrm{f}: \mathrm{E}(\mathrm{G}) \rightarrow\{1,3, \ldots, 2 \mathrm{q}-1\}$ by

$\mathrm{f}\left(\mathrm{e}_{\mathrm{i}}\right)=(2 \mathrm{i}-1)$, for $\mathrm{i}=1,2, \ldots,(3 \mathrm{n}-2) \quad$ (Rule 1$)$

Define $\mathrm{f}_{+}: \mathrm{V}(\mathrm{G}) \rightarrow\{0,1,2, \ldots,(2 \mathrm{k}-1)\}$ by

$\mathrm{f}_{+}(\mathrm{v}) \equiv \Sigma \mathrm{f}(\mathrm{uv}) \bmod (2 \mathrm{k})$, where this sum run over all edges through $v \quad$ (Rule 2).

Hence the map $f$ and the induced map $\quad f_{+}$provide labels as distinct odd numbers for edges and also the labelings for vertex set has distinct values in $\{0,1,2, \ldots,(2 \mathrm{k}-1)\}$. Hence the graph $S_{2} \square S_{n}$, for $n$ is odd, is edge-odd graceful.

Case (ii) : $n$ is even 
Here it is proved that $S_{2} \square S_{n}$ is graceful by taking 2 cases for $\mathrm{n}$ such as

(a). $\mathrm{n} \equiv 2(\bmod 6)$

(b). $\mathrm{n} \equiv 0(\bmod 6)$ and $\mathrm{n} \equiv 4(\bmod 6)$

\section{Subcase $(\mathbf{a}): \mathbf{n} \equiv \mathbf{2}(\bmod 6)$}

Here edges are given labeling with odd numbers as in the figure 3

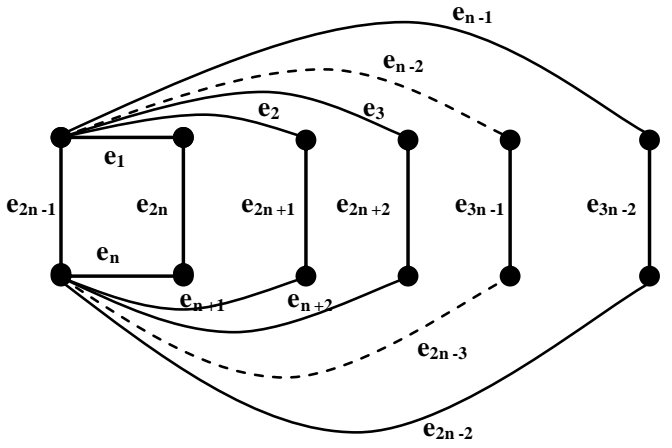

Figure 3: Edge - odd graceful Graph $S_{2} \square S_{n}$, for $\mathbf{n} \equiv \mathbf{2}(\bmod 6)$

Define $f: E(G) \rightarrow\{1,3, \ldots, 2 q-1\}$ by $f\left(e_{i}\right)=(2 i-1)$, for $i=1,2, \ldots,(3 n-2)$ and $\mathrm{i} \neq \mathrm{n}+(\mathrm{n} / 2)-1$ and $2 \mathrm{n}+(\mathrm{n} / 2)-1\}$ (Rule 3$)$ $\mathrm{f}(\mathrm{n}+\mathrm{n} / 2-1)=5 \mathrm{n}-3$ and $f(2 n+n / 2-1)=3 n-3$

Define $\mathrm{f}_{+}: \mathrm{V}(\mathrm{G}) \rightarrow\{0,1,2, \ldots,(2 \mathrm{k}-1)\}$ by $\mathrm{f}_{+}(\mathrm{v}) \equiv \Sigma \mathrm{f}(\mathrm{uv}) \bmod (2 \mathrm{k})$, where this sum run over all edges through $\mathrm{v} \quad$ (Rule 4)

Hence the map $f$ and the induced map $f_{+}$provide labels as distinct odd numbers for edges and also the labelings for vertex set has distinct values in $\{0,1,2, \ldots,(2 \mathrm{k}-1)\}$. Hence the graph $S_{2} \square S_{n}$, for $n \equiv 2(\bmod 6)$, is edge-odd graceful.

\section{Subcase (b): $n \equiv 0,4(\bmod 6)$}

The edges are given labeling as in the figure 4

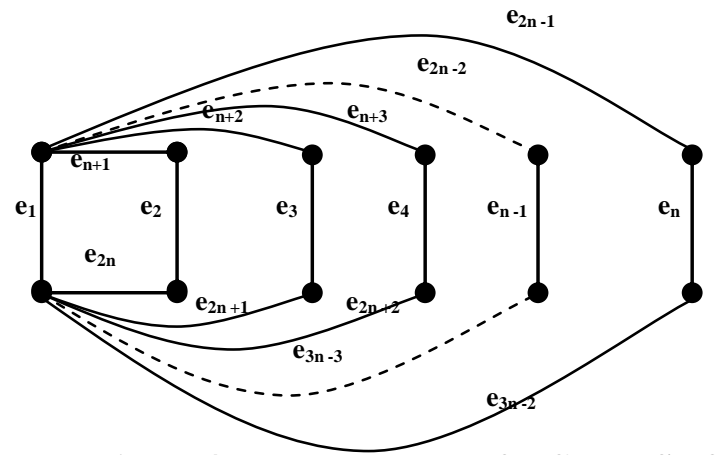

Figure 4: Edge - odd graceful Graph $S_{2} \square S_{n}$, for $n=0,4(\bmod 6)$

Define $f: E(G) \rightarrow\{1,3, \ldots, 2 q-1\}$ by $f\left(e_{i}\right)=(2 i-1)$, for $i=1,2, \ldots,(3 n-2)$

and $\mathrm{i} \neq 1$ and $2 \mathrm{n}-1$ and

For $\mathrm{n} \equiv 0(\bmod 6)$

$f(1)=1$ and $f(2 n-1)=4 n-3$

[Rule 5]

For $\mathrm{n} \equiv 4(\bmod 6)$

$\mathrm{f}(1)=4 \mathrm{n}-3$ and $\mathrm{f}(2 \mathrm{n}-1)=1$

Define $\mathrm{f}_{+}: \mathrm{V}(\mathrm{G}) \rightarrow\{0,1,2, \ldots,(2 \mathrm{k}-1)\}$ by

$\mathrm{f}_{+}(\mathrm{v}) \equiv \Sigma \mathrm{f}(\mathrm{uv}) \bmod (2 \mathrm{k})$, where this sum run over all edges through $\mathrm{v}$

(Rule 6)

Hence the map $\mathrm{f}$ and the induced map $\mathrm{f}_{+}$provide labels as distinct odd numbers for edges and also the labelings for vertex set has distinct values in $\{0,1,2, \ldots,(2 \mathrm{k}-1)\}$. Hence the graph $S_{2} \square S_{n}$, for $n \equiv 0,4(\bmod 6)$, is edge-odd graceful.

Example 2.1: The connected graph $S_{2} \square S_{7}$ is edge - odd graceful.

The following graph (figure 5) is the book graph with 14 vertices and 19 edges, with some arbitrary edge-odd graceful labeling to its vertices and edges.

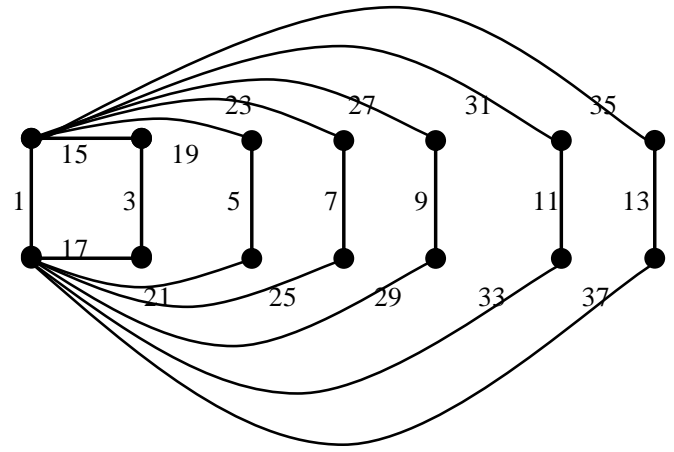

Figure 5: Edge - odd graceful Graph $S_{2} \square S_{7}$ for $n$ odd

Example 2.2: The connected graph $\mathrm{S}_{2} \square \mathrm{S}_{8}$, for $\mathrm{n} \equiv 2(\bmod$ 6 ) is edge - odd graceful.

The figure 6 is the book graph with 16 vertices and 22 edges, with some arbitrary edge-odd graceful labeling to its vertices and edges.

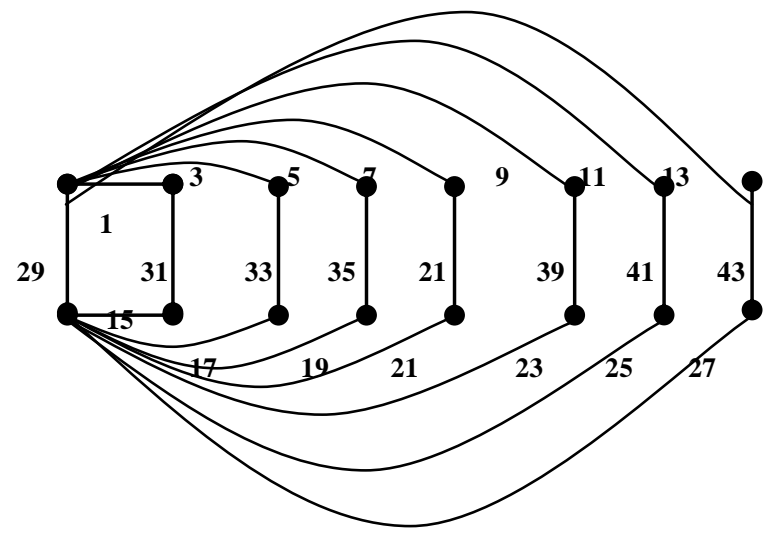

Figure 6: Edge - odd graceful Graph $S_{2} \square S_{8}$ $(\mathrm{n} \equiv 2(\bmod 6))$ 
Example 2.3: The connected graph $\mathrm{S}_{2} \square \mathrm{S}_{6}, \mathrm{~S}_{2} \square \mathrm{S}_{10}(\mathrm{n} \equiv 0,4$ (mod 6)) is edge - odd graceful.

The following graph (figure 7) is the book graph $S_{2} \square S_{6}$ with 12 vertices and 16 edges, with some arbitrary edgeodd graceful labeling to its vertices and edges.

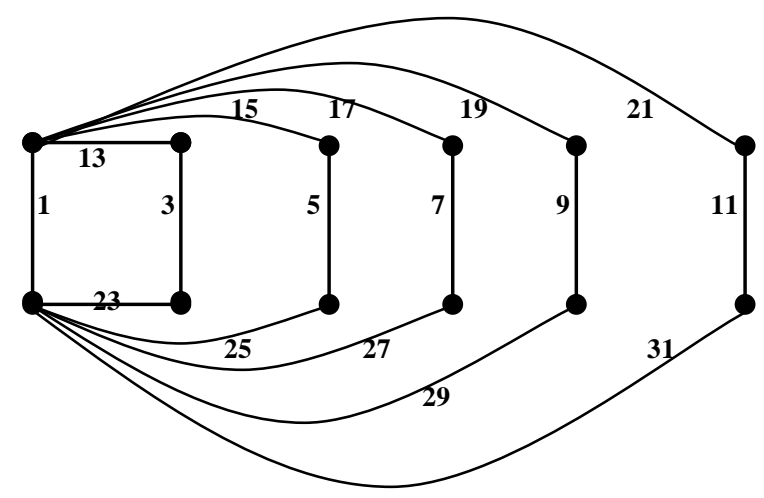

Figure 7: Edge - odd graceful Graph $S_{2} \square S_{6}$, $(\mathbf{n} \equiv \mathbf{0}(\bmod 6))$

The following graph (figure 8) is the book graph $S_{2} \square S_{10}$ with 20 vertices and 28 edges, with some arbitrary edgeodd graceful labeling to its vertices and edges.

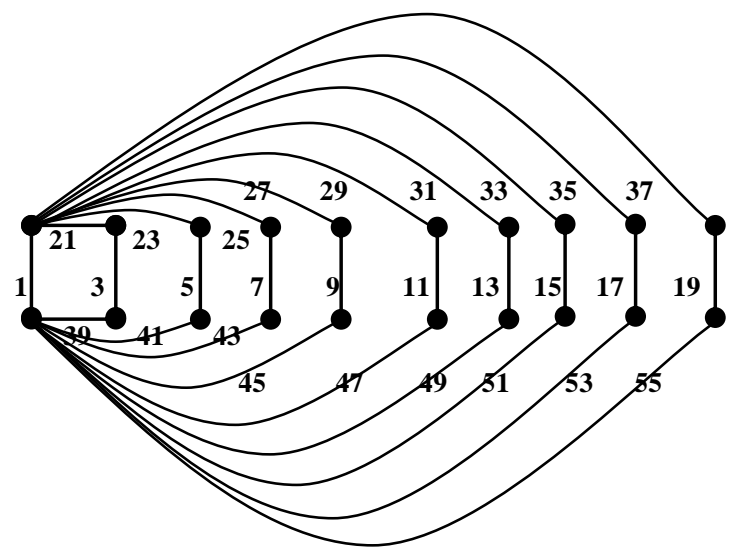

Figure 8: Edge - odd graceful Graph $S_{2} \square S_{10}$, $(n \equiv 4(\bmod 6))$

\section{REFERENCE}

1. A.Solairaju, C.Vimala and A.Sasikala, Gracefulness of a spanning tree of the graph of Cartesian product of $S_{m}$ and $S_{n}$, The Global Journal of Applied Mathematics and Mathematical Sciences, Vol. 1, No-2 (July-Dec 2008): pp 117-120

2. A.Solairaju and K.Chitra, Edge-odd graceful labeling of some graphs, 'Electronics Notes in Discrete Mathematics' Volume 33, April 2009, Pages $15-20$

3. A.Solairaju, A.Sasikala, C.Vimala, 'Edge-odd Gracefulness of a spanning tree of Cartesian product of $\quad \mathrm{P}_{2}$ and $\mathrm{C}_{\mathrm{n}}$,, Pacific-Asian Journal of Mathematics, Vol .3, No. 1-2. Jan-Dec. 2009, pp:3942

4. A.Solairaju, C. Vimala, and A.Sasikala, 'Edgeodd Gracefulness of $\quad C_{3} \quad \Theta \quad P_{n} \quad \& C_{3} \quad \Theta \quad 2 P_{n}$, (communicated to Serial Publications ) 